\title{
mRNA Interferase Bacillus cereus BC0266 Shows MazF-Like Characteristics Through Structural and Functional Study
}

\author{
Sung-Min Kang ${ }^{1,+}{ }^{\oplus}$, Ji Sung Koo ${ }^{1, \dagger}$, Chang-Min Kim ${ }^{1}$, Do-Hee Kim ${ }^{2}$ and Bong-Jin Lee ${ }^{1, *}$ \\ 1 The Research Institute of Pharmaceutical Sciences, College of Pharmacy, Seoul National University, \\ Gwanakgu, Seoul 08826, Korea; men0528@snu.ac.kr (S.-M.K.); koojisung@snu.ac.kr (J.S.K.); \\ kt912@snu.ac.kr (C.-M.K.) \\ 2 College of Pharmacy, Jeju National University, Jeju 63243, Korea; doheekim@jejunu.ac.kr \\ * Correspondence: lbj@nmr.snu.ac.kr; Tel.: +82-2-880-7869 \\ + These authors contributed equally to this work.
}

Received: 15 May 2020; Accepted: 6 June 2020; Published: 8 June 2020

\begin{abstract}
Toxin-antitoxin (TA) systems are prevalent in bacteria and are known to regulate cellular growth in response to stress. As various functions related to TA systems have been revealed, the importance of TA systems are rapidly emerging. Here, we present the crystal structure of putative mRNA interferase BC0266 and report it as a type II toxin MazF. The MazF toxin is a ribonuclease activated upon and during stressful conditions, in which it cleaves mRNA in a sequence-specific, ribosome-independent manner. Its prolonged activity causes toxic consequences to the bacteria which, in turn, may lead to bacterial death. In this study, we conducted structural and functional investigations of Bacillus cereus MazF and present the first toxin structure in the TA system of B. cereus. Specifically, B. cereus MazF adopts a PemK-like fold and also has an RNA substrate-recognizing loop, which is clearly observed in the high-resolution structure. Key residues of B. cereus MazF involved in the catalytic activity are also proposed, and in vitro assay together with mutational studies affirm the ribonucleic activity and the active sites essential for its cellular toxicity.
\end{abstract}

Keywords: toxin-antitoxin system; mazF; type II; toxin; mRNA interferase; X-ray crystallography

Key Contribution: Structural and functional study on putative mRNA interferase BC0266 revealed that $\mathrm{BC} 0266$ is a B. cereus MazF toxin in type II TA system. This is the first reported toxin structure in type II TA system of B. cereus.

\section{Introduction}

Toxin-antitoxin (TA) systems are prevalent in prokaryotes and function to regulate cellular growth in response to stress such as antibiotic exposure, nutrient starvation, heat shock, and DNA damage. Initially, TA systems were discovered as a part of the plasmid maintenance system, in which only the daughter cells harboring the vertically transferred TA operon can survive [1-5]. In addition to the primary maintenance function, TA systems are important for bacterial survival. The TA systems are involved in many cellular processes, including cell growth, cell persistence, cell dormancy, biofilm formation, antibiotic resistance, DNA replication, translation, cell division, cell wall synthesis, and cell apoptosis [6-11].

TA systems are a two-component system, composed of a toxin and an antitoxin usually sharing the same operon. Currently, TA systems can be categorized into six phenotypes (I-VI) by the nature of each component and the regulatory mechanisms of the antitoxins to its cognate toxins. In the case of RNA antitoxins, direct inhibition of mRNA encoding toxins and protein toxins constitute the type I 
and type III systems, respectively. Similarly, protein toxins are directly inhibited by their counterpart protein antitoxins in type II TA system. As for indirect regulatory mechanisms, protein antitoxins counteract the activity of protein toxins in type IV TA system and cleave mRNA encoding protein toxins in type V TA system. Lastly, in type VI TA system, protein toxins are degraded by specific proteases and lose their toxicity as a result of complex formation with protein antitoxins [12-16].

Typically, in type II TA system, the rather stable toxins have much more invariant characteristics than their labile and flexible cognate antitoxins. Upon degradation of antitoxins, free toxins can function in postsegregational cell killing, abortive infection, and even bacterial persistence. Thus, it has been regarded that bacterial persistence would indeed be closely related to type II TA systems due to the cellular process-specific nature of type II toxins [17-21]. One of the best characterized type II toxins is mRNA interferase MazF toxin, belonging to the MazEF family. Comprehensive bioinformatic analyses have shown that MazF is widely distributed in both Gram-positive and Gram-negative bacteria, allowing an in-depth understanding of the consequences of stress and MazF activation on the physiological effects of the bacteria $[6,22,23]$. In addition, understanding of the cutting specificity of MazF on its target RNA is well established. Specifically, the MazF toxin acts as a ribonuclease and cleaves mRNA in a sequence-specific and ribosome-independent manner, but in some cases also cleaves rRNA at specific sequences, exerting its toxicity [17,24,25].

Bacillus cereus is a Gram-positive, facultatively anaerobic, and rod-shaped bacterium that is well known for its association with foodborne illness [26]. Recently, the pathogenicity potential of the bacteria gained attention due to its ability in causing serious and fatal nongastrointestinal infections [27]. As TA systems are involved in several cellular responses such as stress response, bacterial persistence, and biofilm formation, there is a clear linkage between TA systems and the virulence of the bacteria [28]. For this reason, research was conducted on the putative MazF toxin, mRNA interferase BC0266.

Here, we report 2.0 $\AA$ X-ray crystal structure of mRNA interferase MazF as the first toxin structure in the TA system of B. cereus. Similar to that of other MazF toxins, B. cereus MazF adopts a PemK-like fold and also has an elongated loop between the $\beta 1$ and $\beta 2$ strands. Further sequence alignment with several other MazFs supported the key residues in ribonucleic catalysis. Finally, in vitro ribonuclease activity test together with site-directed mutational studies demonstrated that B. cereus MazF exhibits a clear ribonuclease activity and its key residues play a significant role in exerting its role as a ribonuclease. In conclusion, we show that mRNA interferase BC0266 belongs to the MazEF family and is the first reported MazF toxin structure in the TA system of B. cereus. This is an important study to investigate the diversity of the TA system among many different bacterial strains.

\section{Results and Discussion}

\subsection{B. cereus MazF Adopts a PemK-Like Fold}

Crystals of B. cereus MazF were diffracted to high resolution (2.0 $\AA$ ) and the final structure was determined using the molecular replacement method with the MazF structure from Mycobacterium tuberculosis (PDB code 5XE2) [29]. The structure was refined to $R_{\text {work }}$ factor $18.9 \%$ and $R_{\text {free }}$ factor $22.5 \%$. All 116 amino acids were present and lie within the Ramachandran favored region. Crystals had $P 3_{1} 21$ space group with the unit cell dimensions of $a=b=60.648 \AA, c=76.247 \AA$, and $\alpha=\beta=90.0^{\circ}, \gamma=120^{\circ}$. Detailed crystallographic data collection and refinement statistics are summarized in Table 1.

There are three $\alpha$-helices and seven $\beta$-strands with the order of $\beta$-barrel arrangement in a monomer of $B$. cereus MazF (Figure 1A). Among those $\beta$-strands, five $\beta$-strands $(\beta 1, \beta 2, \beta 3, \beta 6, \beta 7)$ and two $\beta$-strands $(\beta 4, \beta 5)$ form a $\beta$-sheet antiparallel to each other. Two loops between the $\beta 1$ and $\beta 2$ strands, and the $\beta 3$ and $\beta 4$ strands are also clearly observed. The total solvent-accessible surface area of the monomeric structure is $6740 \AA^{2}$, and the contact area between two monomers is $1440 \AA^{2}(21.4 \%$ per monomeric subunit). The crystal structure shows that the dimeric interface displays a concave structure covered by the neighboring loop (Figure 1B). Calculations of surface and interface areas were conducted using the PISA server [30]. The oligomeric state of B. cereus MazF was predicted 
through size-exclusion chromatography with reference proteins in the Gel Filtration Calibration Kits (GE Healthcare, Chicago, IL, USA) (Figure 1C). Because theoretical molecular weight of the B. cereus MazF monomer containing N-terminal His-tag is $15.1 \mathrm{kDa}$, it is reasonable that oligomeric state of B. cereus MazF is homodimer $(30.2 \mathrm{kDa})$ as it was eluted at almost the same time as that of carbonic anhydrase $(29 \mathrm{kDa})$.

\subsection{Comparison of B. cereus MazF with MazF Homologs}

To relate the structural characteristics of B. cereus MazF with mRNA interferase MazF, comparative analysis was performed with previously reported MazF structures. Illustrations of sequence alignment were displayed with Staphylococcus aureus MazF (PDB code 4MZM) [31], M. tuberculosis MazF3 (PDB code 5UCT), [32]; MazF4 (PDB code 5XE2) [29]; and MazF7 (PDB code 5WYG) [33] (Figure 2A). The results showed that two key residues of Arg25 and Thr48 involved in catalytic activity are well conserved among multiple MazFs. Interestingly, Lys19 is conserved in M. tuberculosis MazF4 instead of arginine [29], and Ser51 is conserved in M. tuberculosis MazF7 instead of threonine [33]. Yet, mutations of these two residues significantly impaired the activities of corresponding toxins, implying their role in ribonucleic catalysis $[29,33]$. Although considerable structural similarities are shared between the compared MazFs, there are notable differences in flexibilities and structural variations in their $\beta 1-\beta 2$ and $\beta 3-\beta 4$ loops (Figure $2 \mathrm{~B}$ ). These loops are reported as a major binding site with its cognate antitoxin MazE and, therefore, it is supposed that the flexibilities of these loops have relations to interactions with its target RNA substrate depending on MazE binding [32,34,35].

Table 1. Structure data collection and refinement statistics.

\begin{tabular}{|c|c|}
\hline \multicolumn{2}{|c|}{ (a) Data Collection Details } \\
\hline X-ray source & BL44XU beamline of Spring-8, Japan \\
\hline X-ray wavelength $(\AA)$ & 0.899995 \\
\hline Space group & $P 3_{1} 21$ \\
\hline Unit cell parameters: $a, b, c(\AA)$ & $60.648,60.648,76.247$ \\
\hline Unit cell parameters: $\alpha, \beta, \gamma\left({ }^{\circ}\right)$ & $90.0,90.0,120.0$ \\
\hline Resolution range $(\AA)$ & $50.0-2.00$ \\
\hline Observed reflections $(>1 \sigma)$ & 231077 \\
\hline Unique reflections & 21852 \\
\hline$<\mathrm{I} / \sigma(\mathrm{I})>$ & $10.74(2.97) \mathrm{e}$ \\
\hline Completeness (\%) & $99.3(95.5)^{e}$ \\
\hline Multiplicity & $10.57(9.76)^{\mathrm{e}}$ \\
\hline$R_{\text {merge }}(\%)$ & $12.1(47.9)^{\mathrm{e}}$ \\
\hline $\mathrm{CC}_{1 / 2}$ & $0.997(0.918)^{\mathrm{e}}$ \\
\hline \multicolumn{2}{|c|}{ (b) Refinement statistics } \\
\hline$R_{\text {work }}{ }^{\mathrm{c}}(\%)$ & 18.9 \\
\hline$R_{\text {free }} \mathrm{d}(\%)$ & 22.5 \\
\hline No. of atoms/average $B$ factor $\left(\AA^{2}\right)$ & $997 / 46.2$ \\
\hline RMSD $^{\mathrm{f}}$ from ideal geometry: Bond distance $(\AA)$ & 0.008 \\
\hline RMSD $^{\mathrm{f}}$ from ideal geometry: Bond angle $\left(^{\circ}\right)$ & 1.108 \\
\hline Ramachandran statistics: Most favored regions (\%) & 96.49 \\
\hline Ramachandran statistics: Additional allowed regions (\%) & 3.51 \\
\hline PDB accession code & $7 \mathrm{BXY}$ \\
\hline
\end{tabular}

${ }^{\mathrm{a}} N_{\text {obs }} / N_{\text {unique, }}{ }^{\mathrm{b}} R_{\text {merge }}=\Sigma(I-\langle I\rangle) / \Sigma\langle I\rangle,{ }^{\mathrm{c}} R_{\text {work }}=\Sigma_{h k l}|| \mathrm{F}_{\text {obs }}|-k| \mathrm{F}_{\text {calc }}\left|/ \Sigma_{h k l}\right| \mathrm{F}_{\text {obs }} \mid,{ }^{\mathrm{d}} R_{\text {free }}$ was calculated in the same manner as $R_{\text {work }}$ with $5 \%$ of the reflections excluded from the refinement. ${ }^{\text {e }}$ Values in parentheses indicate the highest-resolution shell. ${ }^{\mathrm{f}}$ Root mean square deviation (RMSD) was calculated using REFMAC. 
A
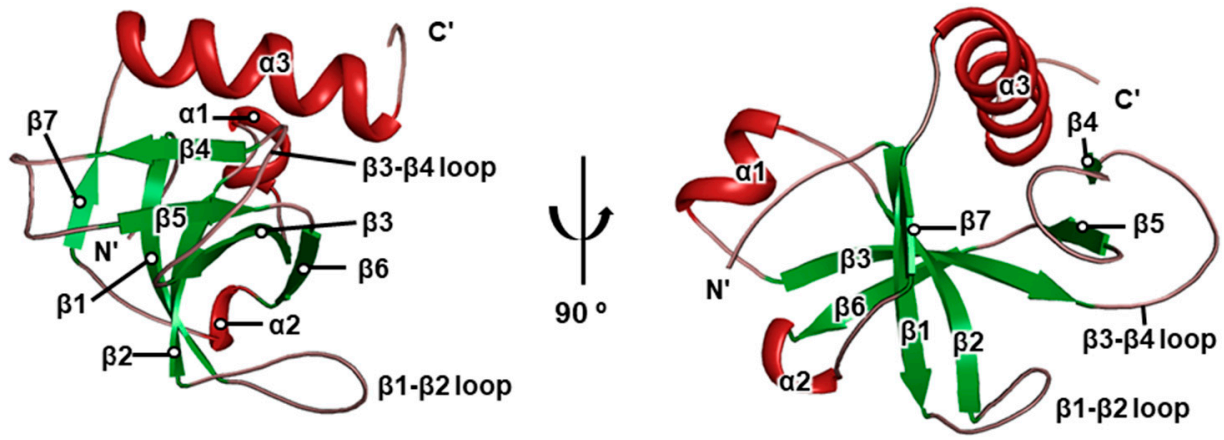

B
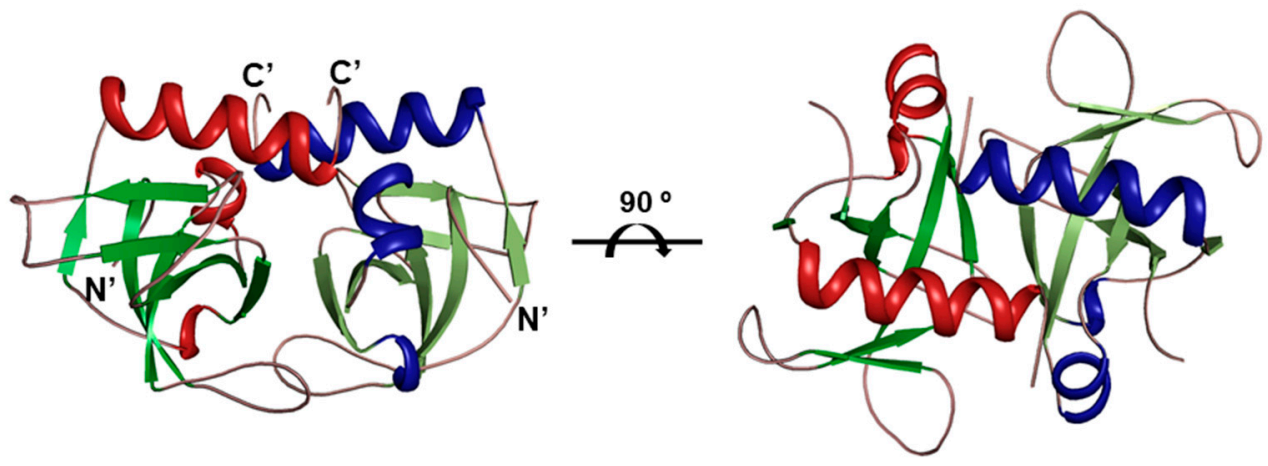

C

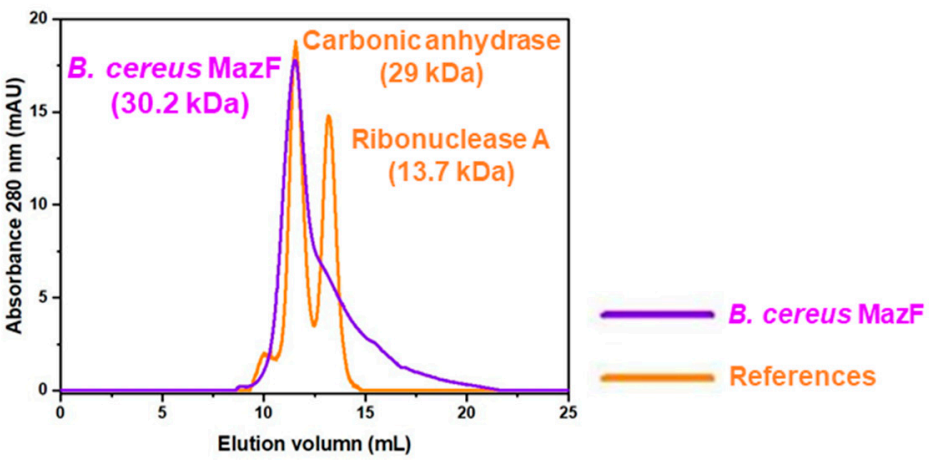

Figure 1. Overall structure of B. cereus MazF and gel filtration data with reference proteins. $\alpha$ helices are colored in red and blue. $\beta$ strands are colored in green. (A) $90^{\circ}$ rotational views on B. cereus MazF monomer. $\beta 1-\beta 2$ and $\beta 3-\beta 4$ loops are denoted. (B) $90^{\circ}$ rotational views on $B$. cereus MazF homodimer. (C) Molecular weight estimates of B. cereus MazF obtained by size-exclusion chromatography with Superdex 75 10/300 gl column. Overlaid chromatograms were denoted with their names and molecular weights. 


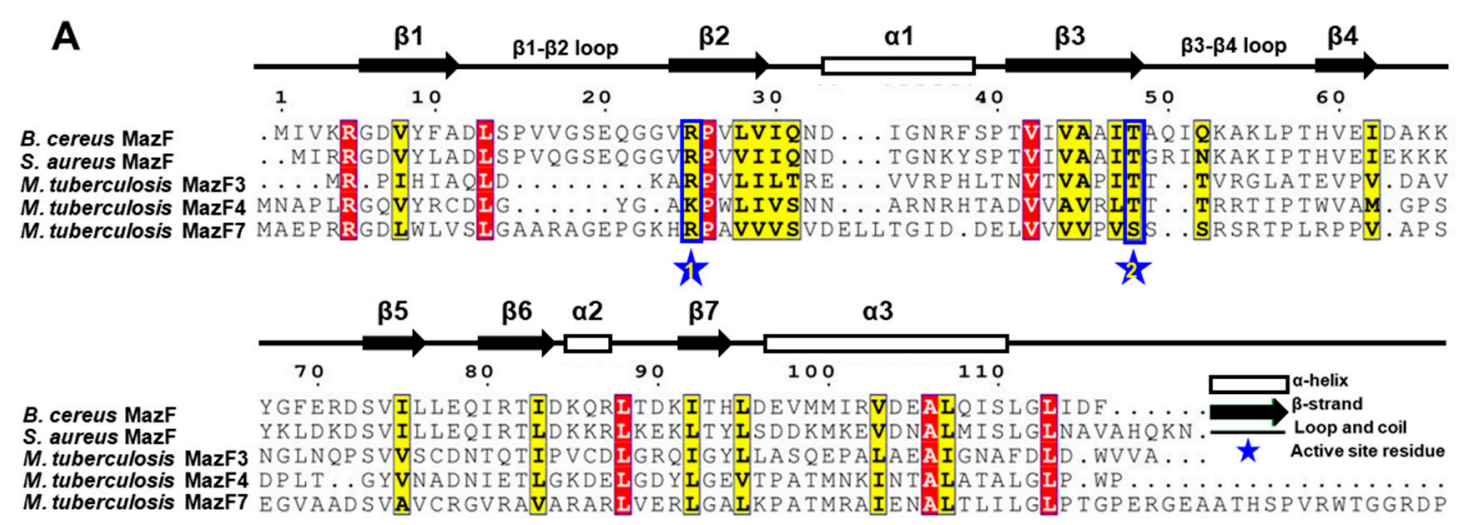

B

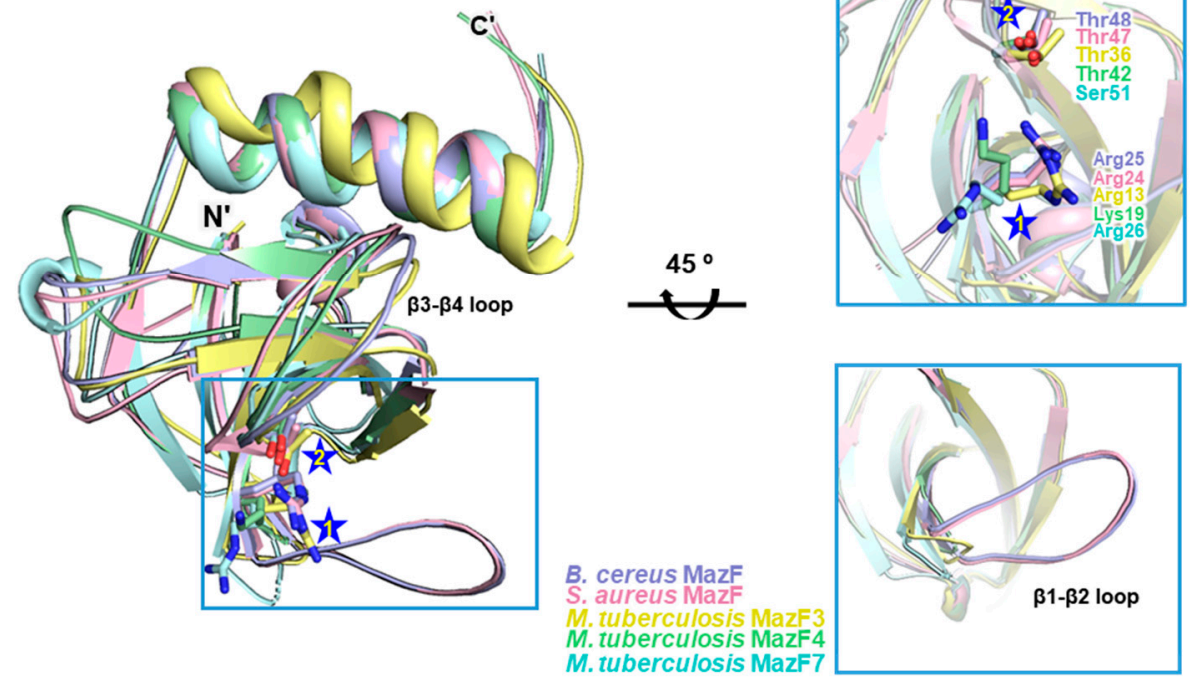

Figure 2. Comparative analysis of MazF toxins. (A) Sequence alignment of B. cereus MazF with other MazFs. Secondary structural elements are displayed above the alignment. Residues showing similarity are highlighted in red and yellow. Conserved active site residues are emphasized with star symbol. (B) Structural comparison of B. cereus MazF with other MazFs. Cartoon representations are employed to draw each structures. Conserved active site residues are shown in sticks. Conserved active site residues and variations in $\beta 1-\beta 2$ loop are illustrated by enlarged view in blue square.

Through early studies on E. coli MazEF, it was suggested that the $\beta 1-\beta 2$ loop in the MazF toxin undergoes conformational transition from disordered to ordered state upon binding to the MazE antitoxin [36]. During this transition, it is assumed that these loops are modulated by MazE, affecting the target RNA accessibility of MazF [36]. For instance, MazF4 and MazF7 toxins from M. tuberculosis with truncated $\beta 1-\beta 2$ loops had significant steric clashes within the $\beta 1-\beta 2$ loop region due to the high flexibility arising from the structurally unbound MazE antitoxin [29,33]. As for MazF3 toxin in $M$. tuberculosis, the length of the short $\beta 1-\beta 2$ loop may be insufficient to reach and recognize the downstream of the target RNA substrate [31,32]. Furthermore, it is predicted that absence of an elongated $\beta 1-\beta 2$ loop would cause even greater conformational transition of the $\beta 1-\beta 2$ loop, leaving the toxin structurally and functionally impractical. Therefore, it is suggested that target sequence specificity of truncated or short $\beta 1-\beta 2$ loops would be significantly reduced compared with MazFs having a long $\beta 1-\beta 2$ loop, such as that of $B$. cereus MazF. Therefore, it can be presumed that the long length of $\beta 1-\beta 2$ loop may be the most powerful factor that determines the target RNA specificity.

In contrast to the $\beta 1-\beta 2$ loop, $\beta 3-\beta 4$ loop shows an opposite conformational transition from ordered to disordered state upon binding to MazE antitoxin [29]. Furthermore, previous research has shown that aromatic residues located on the antitoxin MazEs are involved in essential MazEF 
interactions [29]. Specifically, His68 of E. coli MazE, Tyr61 of B. subtilis MazE, and Tyr76 of M. tuberculosis MazE4 interacted with the $\beta 3-\beta 4$ loops via van der Walls interactions $[29,34,35]$.

Thus, considering the E. coli and M. tuberculosis model proteins, the B. cereus MazE antitoxin, $\mathrm{BC} 0265$, likely contains $C$-terminal aromatic residues that might interact with the $\beta 3-\beta 4$ loop in $B$. cereus MazF. Because of high structural unpredictability and lack of structural information of $B$. cereus MazE and other MazE antitoxins in general, an accurate prediction of which aromatic residue will interact with its cognate toxin is difficult. However, assuming the model is correct, one of the aromatic residues in B. cereus MazE, Tyr63, Phe78, Tyr82 or His86, likely engages in a hydrophobic interaction during complex formation.

\subsection{Arg25 and Thr48 Act as Key Residues in Catalytic Activity}

To assure that two key residues Arg25 and Thr48 are critical in catalytic activity, comparative analysis with B. subtilis MazF complexed with RNA (PDB code 4MDX) [34] (Figure 3A) and E. coli MazF complexed with DNA substrate analog (PDB code 5CR2) [35] (Figure 3B) was performed. Subsequently, in silico model of B. cereus MazF complexed with RNA and DNA was generated by superimposition of B. cereus MazF structure onto the 4MDX (left) and 5CR2 (right), respectively (Figure 3C).

A

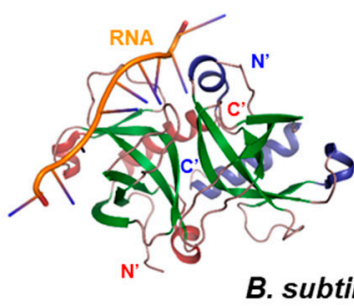

B. subtilis MazF

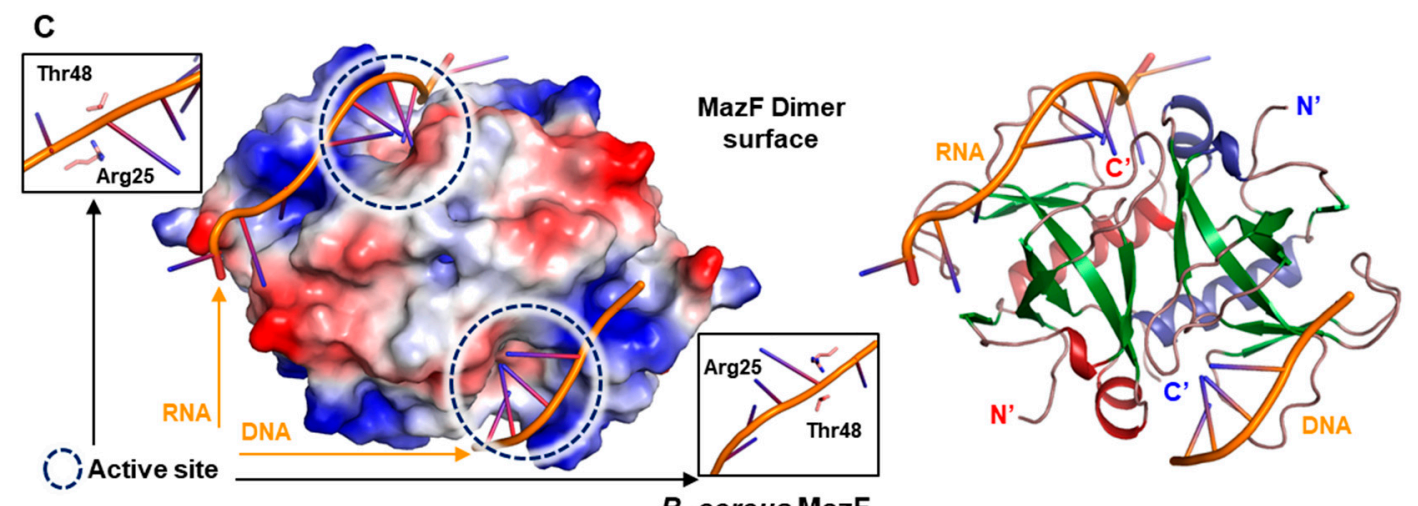

Figure 3. In silico model of previously reported MazFs and B. cereus MazF in complex with RNA and DNA. (A-C) Active sites for each proteins are illustrated in circles. (A) Ribbon representations and electrostatic surface potentials of B. subtilis MazF complexed with RNA. RNA is handled to be placed at left chain. (B) Ribbon representations and electrostatic surface potentials of E. coli MazF complexed with DNA. DNA is handled to be placed at right chain. (C) Superimposition of B. cereus MazF on $B$. subtilis MazF complexed with RNA (left chain) and E. coli MazF complexed with DNA (right chain). Close-up views of the interactions between active site residues and RNA/DNA are displayed.

In the in silico model of B. cereus MazF in complex with RNA and DNA substrate, Arg25 and Thr48 residues were well organized with RNA and DNA substrate (Figure 3C). Moreover, mutational study on B. subtilis MazF Arg25 and Thr48 resulted in complete inactivation of toxicity of MazF [34]. From these results, it can be assumed that Arg25 and Thr48 from B. cereus would also be involved in RNA catalysis. In addition, according to earlier work performed on MazF toxins, Arg25 is predicted to 
act as a general acid/base during RNA catalysis and Thr48 may serve as a charge stabilizer in transition state during RNA substrate binding [35,37,38].

Interestingly, charge distributions observed in the front surfaces of three proteins (Figure $3 \mathrm{~A}-\mathrm{C}$ ) are similar but slightly different from each other, mainly due to the differences in the charged amino acid content. In E. coli MazF, negative-charged front surface is mostly formed by Asp16, Asp18, and Glu24 in the $\beta 1-\beta 2$ loop (residues 16 to 27). However, corresponding residues are substituted with different amino acids in both B. subtilis and B. cereus MazF. Because these front surfaces are known as the binding region of cognate antitoxin MazE, each MazE is thought to have a surface potential specific to its cognate MazF toxin to effectively bind and neutralize toxin surfaces. This seems to be the one of strong reasons why a specific toxin is neutralized only by its cognate antitoxin, although considerable structural similarities are observed within the same family of TA system $[29,39,40]$.

\subsection{B. cereus MazF Shows Ribonuclease Activity by Two Key Residues}

MazF is a ribonuclease that is known to cleave RNA substrate [41]. Toxins such as VapC in the Type II TA system require metal ions as a cofactor to exhibit ribonuclease activity, but in the case of MazF, cofactor metal is not necessarily needed [42,43]. To demonstrate that B. cereus MazF shows ribonuclease activity like other MazF toxins, in vitro ribonuclease activity test was performed (Figure 4A). In this assay, a synthetic RNA strand of unknown sequence is used as substrate. A fluorophore is covalently attached to one end of the RNA strand while the quencher is located at the other end. If this RNA is cleaved by a ribonuclease, the quencher is detached and as a result fluorescence is detected depending on the amount of cleaved RNA. The result from in vitro ribonuclease assay showed an elevation of RFU (resulting fluorescence unit) as a function of time. B. cereus MazF exhibits a ribonuclease activity showing time and dose dependency, and hence shares the same functional activity with MazF toxins.
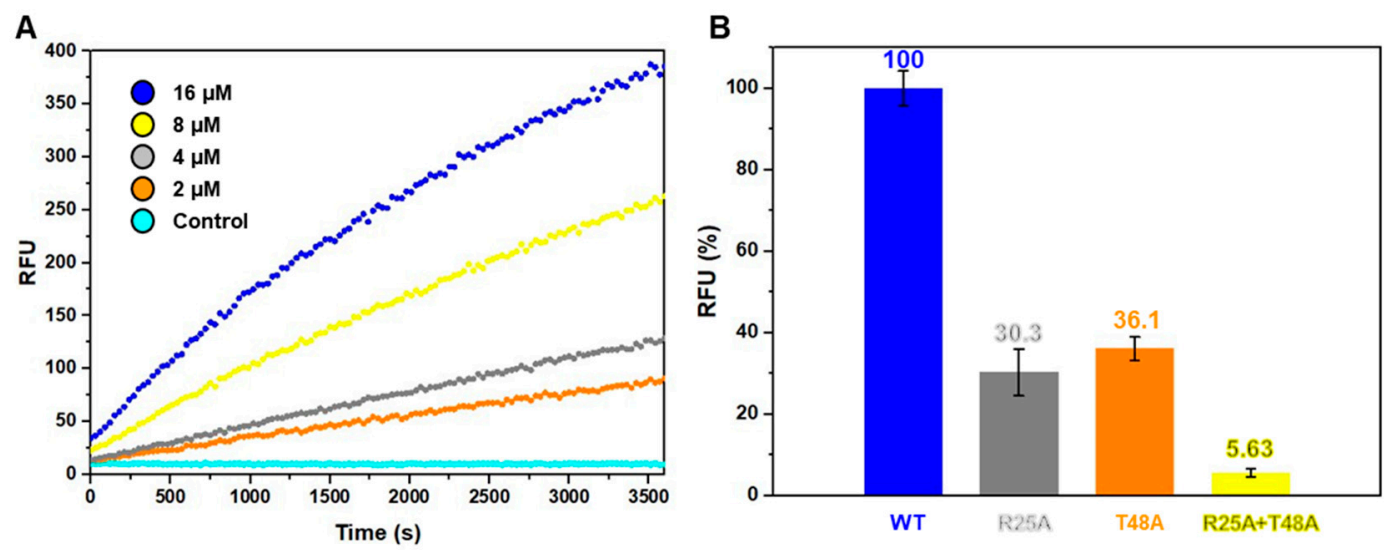

Figure 4. Ribonuclease activity test of B. cereus MazF toxin. (A) The in vitro ribonuclease activity test of wild-type B. cereus MazF. Fluorescence was measured as a function of time (s) during $1 \mathrm{~h}$. Each curve is colored separately according to different B. cereus MazF concentrations. Concentrations of B. cereus $\mathrm{MazF}$ were increased by doubling from 2 to $16 \mu \mathrm{M}$. An equal aliquot of fluorescent RNA substrate was incubated with B. cereus MazF in different concentrations. Fluorescence was measured upon cleavage of RNA substrate. The control as well as different protein concentrations contained $20 \mathrm{mM}$ Tris- $\mathrm{HCl}$ (pH 8.0), $500 \mathrm{mM} \mathrm{NaCl}$, and 40 units of RiboLock ${ }^{\mathrm{TM}}$ (Thermo Scientific, Waltham, MA, USA) RNase inhibitor. (B) Ribonuclease activities of $10 \mu \mathrm{M}$ wild-type B. cereus MazF and its mutants represented as bar graph with standard deviation derived from triplicate tests. Magnitudes of each reaction were assessed by subtracting the RFU (resulting fluorescence unit) of the starting point from RFU of the end point. The RFU obtained from wild-type B. cereus MazF was taken as $100 \%$.

Furthermore, two single and one double site-directed mutagenesis on the two key catalytic residues of Arg25 and Thr48 were performed (Figure 4B). The results of the mutational studies showed that single mutations of either Arg25 or Thr48 resulted in severe reductions in the ribonuclease activity 
of toxin while double mutations of both Arg25 and Thr48 resulted in close to none ribonuclease activity. Therefore, in agreement with the results of B. subtilis MazF, Arg25 and Thr48 are key residues in B. cereus MazF, which is a characteristic observed consistently in MazF toxins.

\section{Conclusions}

We conducted an in-depth characterization and investigation on putative mRNA interferase MazF in $B$. cereus. High-resolution $(2.0 \AA)$ structure was obtained by X-ray crystallography, and overall structural folding of $B$. cereus MazF indicated $\beta$-barrel arrangement containing two independent $\beta$-sheets identical to that of MazF toxins. Through comparison analysis with previously reported structures of MazFs, amino acid residues Arg25 and Thr48 in B. cereus MazF were well conserved with other MazFs and were also confirmed as key residues in the catalytic activity of B. cereus MazF. Also, $\beta 1-\beta 2$ and $\beta 3-\beta 4$ loops were clearly observed in B. cereus MazF structure and their role in the recognition of target RNA substrate depending on the binding of its cognate antitoxin was discussed. From previously conducted studies, it can be presumed that the $\beta 1-\beta 2$ loop acts as a gateway to binding of its target RNA substrate and cognate antitoxin and the $\beta 3-\beta 4$ loop also acts as a major interaction site in antitoxin-binding via van der Walls interactions. Lastly, ribonuclease activity test via in vitro assay together with site-directed mutational studies on Arg25 and Thr48 showed a decrease in its ribonuclease activity, which was consistent in other MazF toxins. Altogether, our study provides a unified structural and functional basis that mRNA interferase BC0266 is indeed a MazF toxin. This is the first determined MazF and toxin structure in type II TA system of B. cereus. Our work may be a rational basis for understanding the TA systems regarding the stress-responsive toxin MazF and the general regulatory mechanisms of TA systems.

\section{Materials and Methods}

\subsection{Cloning and Transformation}

The $B C 0266$ gene was amplified by polymerase chain reaction (PCR). The following primers were used in PCR: forward, 5'- GGAATTCCATATGATGATTGTAAAACGCGGC-3'; reverse, 5' CCGCTCGAGTTAAAAATCTATTAGTCCTAAAC-3' . Nde1 and Xho1 were used as restriction enzymes, and the cutting sites are underlined. The PCR products and pET28a vector were double-cleaved by the same restriction enzymes and ligated to each other. For purification, residual $\mathrm{N}$-terminal His tag (MGSSHHHHHHSSGLVPRGSH) was attached to the gene of B. cereus MazF and was transformed into E.coli DH5 $\alpha$ competent cells (Novagen, Madison, WI, USA). As the next steps, these cloned plasmids were re-transformed into E. coli Rosetta2 (DE3) pLysS competent cells (Novagen, Madison, WI, USA).

\subsection{Protein Expression and Purification}

Transformed cells were grown in Luria broth (LB) at $37^{\circ} \mathrm{C}$ until the $\mathrm{OD}_{600}$ reached to 0.5 . Overexpression of target protein was induced by $0.5 \mathrm{mM}$ isopropyl 1-thio-B-D-galactopyranoside (IPTG). Expressed cells were further incubated for $4 \mathrm{~h}$ at $37^{\circ} \mathrm{C}$. These cells were harvested by centrifugation at $11,355 \times \mathrm{g}$. Harvested cells were suspended in a buffer A ( $20 \mathrm{mM}$ Tris- $\mathrm{HCl}, \mathrm{pH} 7.9$, and $500 \mathrm{mM} \mathrm{NaCl}$ ) containing $5 \%$ glycerol and lysed by ultrasonication. Lysed cells were centrifugated for $1 \mathrm{~h}$ at $28,306 \times \mathrm{g}$. After centrifugation, the supernatant containing soluble moiety of protein was loaded on a $\mathrm{Ni}^{2+}$ affinity chromatography column (Bio-Rad, Hercules, CA, USA), which was pre-equilibrated with buffer A. Loaded protein was washed with buffer A containing $50 \mathrm{mM}$ imidazole to remove impurities. Then, the remaining protein, bound to the $\mathrm{Ni}^{2+}$ column, was eluted using an imidazole gradient (100-800 $\mathrm{mM}$ ) and fractionized. The purity of eluted protein in each fraction was checked using sodium dodecyl sulfate polyacrylamide gel electrophoresis (SDS-PAGE). Finally, selected high-purity protein was loaded on a size-exclusion chromatography column HiLoad 16/600 Superdex 200 prep-grade column (GE Healthcare, Chicago, IL, USA), pre-equilibrated with final buffer ( $20 \mathrm{mM}$ Tris, $\mathrm{pH} 8.0$, and $500 \mathrm{mM} \mathrm{NaCl}$ ). Final eluted protein was concentrated to $20 \mathrm{mg} / \mathrm{mL}$ using an 
Amicon Ultra centrifugal filter unit (Millipore, Burlington, MA, USA). The purity of the final protein solution was verified by SDS-PAGE. Mutants of B. cereus MazF were expressed and purified by the same procedure as native ones.

\subsection{Crystallization, Data Collection, and Processing}

The final protein solution was screened for crystallization using crystallization kit Wizard (Rigaku Reagents, Bainbridge Island, WA, USA) and Index (Hampton Research, Aliso Viejo, CA, USA). Protein solution $(1 \mu \mathrm{L})$ was mixed with $1 \mu \mathrm{L}$ of each buffer solution in the crystallization kit. Crystals of $B$. cereus MazF were grown in the transparent 96-well plate using sitting-drop vapor diffusion method at $20^{\circ} \mathrm{C}$. Only one crystallized well contained the hit solution of $100 \mathrm{mM}$ Sodium Citrate, $\mathrm{pH} 5.6$, and $1.0 \mathrm{M}$ ammonium phosphate monobasic. Cryo-protection was achieved by addition of $20 \%$ glycerol to the hit solution. After cryo-protection, crystals were immediately cooled in liquid nitrogen prior to data collection. The data were collected using an Rayonix MX-300 HE CCD detector at BL44XU of the Spring-8, Japan. All raw data of crystal were scaled and processed by XDS [44]. A set of data from a hit crystal was used to solve the structure at $2.00 \AA$ resolution and to refine 7BXY (code name of B. cereus MazF). PHENIX [45] was first used to do molecular replacement and automatically build the model, and COOT [46] was utilized to yield the starting model for refinement. The $R_{\text {work }} / R_{\text {free }}$ values [47] of the final model were obtained using REFMAC and PHENIX [45,48]. The validation of overall geometry was achieved using MolProbity [49]. PyMOL (PyMOL Molecular Graphics System, Version 1.2r3pre, Schrödinger, LLC, NY, USA) was used to generate figures in this study.

\subsection{Mutiple Sequence Alignment}

To conduct sequence alignment of B. cereus MazF and four MazFs whose structures have been identified, the sequence information of five proteins was browsed using Uniprot [50]. Alignments of amino acid residues were carried out using Clustal W [51] and visualized using ESPript 3.0 [52]. The consensus value was set to 0.85 and \%Equivalent was used in similarity mode. In visualization, structural information of B. cereus MazF was used as top secondary structures and the description and sequence numbering on the topside correspond to B. cereus MazF.

\subsection{In Vitro Ribonuclease Assay}

To confirm the ribonuclease activity of B. cereus MazF, an RNase Alert Kit (IDT, Coralville, IA, USA) was purchased and used following the manufacturer's protocol. Equal aliquot RNA substrate $(5 \mu \mathrm{L})$ was interacted with $2 \mu \mathrm{M}, 4 \mu \mathrm{M}, 8 \mu \mathrm{M}$, and $16 \mu \mathrm{M}$ concentrations of B. cereus MazF in a final purification buffer. For ribonuclease activity test, $10 \mu \mathrm{M}$ aliquots of wild-type B. cereus MazF and its mutants were prepared. Then, the resulting fluorescence units (RFU) were detected using a SPECTRAmax GEMINI XS spectro-fluorometer (Marshall Scientific, Hampton, NH, USA) at $37^{\circ} \mathrm{C}$ by emission fluorescence at $520 \mathrm{~nm}$ upon excitation at $490 \mathrm{~nm}$. Assay setting was performed on 384-well opaque plate, and all of experiments were performed in triplicate.

\subsection{Site-Directed Mutagenesis}

The mutations in B. cereus MazF (R25A, T48A, R25A+T48A) were performed using B. cereus MazF in pET28a (+) as template. Single-site mutations were performed in one step, and double-site mutations were performed in a step-wise manner. Reaction components were mixed and subjected to PCR machine guided by the manufacturer's protocol (EZchange Site-Directed Mutagenesis Kit, Enzynomics, Daejeon, Korea). Each plasmid was transformed into E. coli XL10-Gold competent cells (Agilent Technologies, Santa Clara, CA, USA), and the resulting inserted genes were verified through DNA sequencing.

Author Contributions: Conceptualization, S.-M.K. and J.S.K.; Methodology, S.-M.K. and J.S.K.; Data curation, S.-M.K. and J.S.K.; Formal analysis, S.-M.K., J.S.K and C.-M.K.; Writing, original draft preparation, S.-M.K., J.S.K. 
and D.-H.K.; Writing: review and editing, S.-M.K., J.S.K., D.-H.K and B.-J.L.; All the authors approved the final version of the manuscript. All authors have read and agreed to the published version of the manuscript.

Funding: This research was funded by a National Research Foundation of Korea (NRF) grant funded by the Korean government (grant numbers 2018R1A2A1A19018526, 2018R1A5A2024425, 2019R1C1C1002128, and 2019R1I1A1A01057713) and the 2019 BK21 Plus Project for Medicine, Dentistry and Pharmacy. This work was also funded by International Program (ICR-20-05) of IPR, Osaka University.

Acknowledgments: We thank the beamline (BL) staff members at Pohang Light Source (BL-5C, BL-7A and BL11C), Korea, and SPring-8 (BL44XU) under the Collaborative Research Program of Institute for Protein Research, Osaka University (proposal No. 2019A6972).

Conflicts of Interest: The authors declare they have no conflict of interest.

\section{References}

1. Page, R.; Peti, W. Toxin-antitoxin systems in bacterial growth arrest and persistence. Nat. Chem. Biol. 2016, 12, 208-214. [CrossRef]

2. Yamaguchi, Y.; Park, J.H.; Inouye, M. Toxin-antitoxin systems in bacteria and archaea. Annu. Rev. Genet. 2011, 45, 61-79. [CrossRef] [PubMed]

3. Lee, K.Y.; Lee, B.J. Structure, Biology, and Therapeutic Application of Toxin-Antitoxin Systems in Pathogenic Bacteria. Toxins 2016, 8, 305. [CrossRef] [PubMed]

4. Ogura, T.; Hiraga, S. Mini-F plasmid genes that couple host cell division to plasmid proliferation. Proc. Natl. Acad. Sci. USA 1983, 80, 4784-4788. [CrossRef] [PubMed]

5. Jurenaite, M.; Markuckas, A.; Suziedeliene, E. Identification and Characterization of Type II Toxin-Antitoxin Systems in the Opportunistic Pathogen Acinetobacter baumannii. J. Bacteriol. 2013, 195, 3165-3172. [CrossRef]

6. Pandey, D.P.; Gerdes, K. Toxin-antitoxin loci are highly abundant in free-living but lost from host-associated prokaryotes. Nucleic Acids Res. 2005, 33, 966-976. [CrossRef]

7. Sengupta, M.; Austin, S. Prevalence and significance of plasmid maintenance functions in the virulence plasmids of pathogenic bacteria. Infect. Immun. 2011, 79, 2502-2509. [CrossRef]

8. Kwan, B.W.; Valenta, J.A.; Benedik, M.J.; Wood, T.K. Arrested protein synthesis increases persister-like cell formation. Antimicrob. Agents Chemother. 2013, 57, 1468-1473. [CrossRef]

9. Goeders, N.; Van Melderen, L. Toxin-Antitoxin Systems as Multilevel Interaction Systems. Toxins 2014, 6, 304-324. [CrossRef]

10. Mutschler, H.; Gebhardt, M.; Shoeman, R.L.; Meinhart, A. A novel mechanism of programmed cell death in bacteria by toxin-antitoxin systems corrupts peptidoglycan synthesis. PLoS Biol. 2011, 9, e1001033. [CrossRef]

11. Unoson, C.; Wagner, E.G.H. A small SOS-induced toxin is targeted against the inner membrane in Escherichia coli. Mol. Microbiol. 2008, 70, 258-270. [CrossRef]

12. Goeders, N.; Chai, R.; Chen, B.H.; Day, A.; Salmond, G.P.C. Structure, Evolution, and Functions of Bacterial Type III Toxin-Antitoxin Systems. Toxins 2016, 8, 282. [CrossRef]

13. Lobato-Marquez, D.; Diaz-Orejas, R.; Garcia-del Portillo, F. Toxin-antitoxins and bacterial virulence. FEMS Microbiol. Rev. 2016, 40, 592-609. [CrossRef]

14. Harms, A.; Brodersen, D.E.; Mitarai, N.; Gerdes, K. Toxins, Targets, and Triggers: An Overview of Toxin-Antitoxin Biology. Mol. Cell 2018, 70, 768-784. [CrossRef]

15. Wang, X.X.; Lord, D.M.; Cheng, H.Y.; Osbourne, D.O.; Hong, S.H.; Sanchez-Torres, V.; Quiroga, C.; Zheng, K.; Herrmann, T.; Peti, W.; et al. A new type V toxin-antitoxin system where mRNA for toxin GhoT is cleaved by antitoxin GhoS. Nat. Chem. Biol. 2012, 8, 855-861. [CrossRef]

16. Aakre, C.D.; Phung, T.N.; Huang, D.; Laub, M.T. A Bacterial Toxin Inhibits DNA Replication Elongation through a Direct Interaction with the beta Sliding Clamp. Mol. Cell 2013, 52, 617-628. [CrossRef] [PubMed]

17. Yamaguchi, Y.; Inouye, M. mRNA interferases, sequence-specific endoribonucleases from the toxin-antitoxin systems. Prog. Mol. Biol. Transl. Sci. 2009, 85, 467-500. [CrossRef] [PubMed]

18. Aizenman, E.; Engelberg-Kulka, H.; Glaser, G. An Escherichia coli chromosomal "addiction module" regulated by guanosine [corrected] 3',5'-bispyrophosphate: A model for programmed bacterial cell death. Proc. Natl. Acad. Sci. USA 1996, 93, 6059-6063. [CrossRef] [PubMed]

19. Marianovsky, I.; Aizenman, E.; Engelberg-Kulka, H.; Glaser, G. The regulation of the Escherichia coli mazEF promoter involves an unusual alternating palindrome. J. Boil. Chem. 2001, 276, 5975-5984. [CrossRef] [PubMed] 
20. Kedzierska, B.; Lian, L.Y.; Hayes, F. Toxin-antitoxin regulation: Bimodal interaction of YefM-YoeB with paired DNA palindromes exerts transcriptional autorepression. Nucleic Acids Res. 2007, 35, 325-339. [CrossRef]

21. Brantl, S.; Muller, P. Toxin(-)Antitoxin Systems in Bacillus subtilis. Toxins 2019, 11, 262. [CrossRef] [PubMed]

22. Leplae, R.; Geeraerts, D.; Hallez, R.; Guglielmini, J.; Dreze, P.; Van Melderen, L. Diversity of bacterial type II toxin-antitoxin systems: A comprehensive search and functional analysis of novel families. Nucleic Acids Res. 2011, 39, 5513-5525. [CrossRef] [PubMed]

23. Anantharaman, V.; Aravind, L. New connections in the prokaryotic toxin-antitoxin network: Relationship with the eukaryotic nonsense-mediated RNA decay system. Genome Biol. 2003, 4, R81. [CrossRef] [PubMed]

24. Schifano, J.M.; Edifor, R.; Sharp, J.D.; Ouyang, M.; Konkimalla, A.; Husson, R.N.; Woychik, N.A. Mycobacterial toxin MazF-mt6 inhibits translation through cleavage of $23 \mathrm{~S}$ rRNA at the ribosomal A site. Proc. Natl. Acad. Sci. USA 2013, 110, 8501-8506. [CrossRef] [PubMed]

25. Vesper, O.; Amitai, S.; Belitsky, M.; Byrgazov, K.; Kaberdina, A.C.; Engelberg-Kulka, H.; Moll, I. Selective translation of leaderless mRNAs by specialized ribosomes generated by MazF in Escherichia coli. Cell 2011, 147, 147-157. [CrossRef] [PubMed]

26. Ehling-Schulz, M.; Lereclus, D.; Koehler, T.M. The Bacillus cereus Group: Bacillus Species with Pathogenic Potential. Microbiol. Spectr. 2019, 7. [CrossRef] [PubMed]

27. Bottone, E.J. Bacillus cereus, a volatile human pathogen. Clin. Microbiol. Rev. 2010, 23, 382-398. [CrossRef] [PubMed]

28. Fernandez-Garcia, L.; Blasco, L.; Lopez, M.; Bou, G.; Garcia-Contreras, R.; Wood, T.; Tomas, M. Toxin-Antitoxin Systems in Clinical Pathogens. Toxins 2016, 8, 227. [CrossRef]

29. Ahn, D.H.; Lee, K.Y.; Lee, S.J.; Park, S.J.; Yoon, H.J.; Kim, S.J.; Lee, B.J. Structural analyses of the MazEF4 toxin-antitoxin pair in Mycobacterium tuberculosis provide evidence for a unique extracellular death factor. J. Biol. Chem. 2017, 292, 18832-18847. [CrossRef]

30. Krissinel, E.; Henrick, K. Inference of macromolecular assemblies from crystalline state. J. Mol. Biol. 2007, 372, 774-797. [CrossRef]

31. Zorzini, V.; Buts, L.; Sleutel, M.; Garcia-Pino, A.; Talavera, A.; Haesaerts, S.; De Greve, H.; Cheung, A.; van Nuland, N.A.; Loris, R. Structural and biophysical characterization of Staphylococcus aureus SaMazF shows conservation of functional dynamics. Nucleic Acids Res. 2014, 42, 6709-6725. [CrossRef]

32. Hoffer, E.D.; Miles, S.J.; Dunham, C.M. The structure and function of Mycobacterium tuberculosis MazF-mt6 toxin provide insights into conserved features of MazF endonucleases. J. Biol. Chem. 2017, 292, 7718-7726. [CrossRef]

33. Chen, R.; Tu, J.; Liu, Z.; Meng, F.; Ma, P.; Ding, Z.; Yang, C.; Chen, L.; Deng, X.; Xie, W. Structure of the MazF-mt9 toxin, a tRNA-specific endonuclease from Mycobacterium tuberculosis. Biochem. Biophys. Res. Commun. 2017, 486, 804-810. [CrossRef]

34. Simanshu, D.K.; Yamaguchi, Y.; Park, J.H.; Inouye, M.; Patel, D.J. Structural basis of mRNA recognition and cleavage by toxin MazF and its regulation by antitoxin MazE in Bacillus subtilis. Mol. Cell 2013, 52, 447-458. [CrossRef]

35. Zorzini, V.; Mernik, A.; Lah, J.; Sterckx, Y.G.; De Jonge, N.; Garcia-Pino, A.; De Greve, H.; Versees, W.; Loris, R. Substrate Recognition and Activity Regulation of the Escherichia coli mRNA Endonuclease MazF. J. Biol. Chem. 2016, 291, 10950-10960. [CrossRef] [PubMed]

36. Kamada, K.; Hanaoka, F.; Burley, S.K. Crystal structure of the MazE/MazF complex: Molecular bases of antidote-toxin recognition. Mol. Cell 2003, 11, 875-884. [CrossRef]

37. Guillen Schlippe, Y.V.; Hedstrom, L. A twisted base? The role of arginine in enzyme-catalyzed proton abstractions. Arch. Biochem. Biophys. 2005, 433, 266-278. [CrossRef] [PubMed]

38. Dunican, B.F.; Hiller, D.A.; Strobel, S.A. Transition State Charge Stabilization and Acid-Base Catalysis of mRNA Cleavage by the Endoribonuclease RelE. Biochemistry 2015, 54, 7048-7057. [CrossRef] [PubMed]

39. Gogos, A.; Mu, H.; Bahna, F.; Gomez, C.A.; Shapiro, L. Crystal structure of YdcE protein from Bacillus subtilis. Proteins 2003, 53, 320-322. [CrossRef] [PubMed]

40. Kang, S.M.; Kim, D.H.; Jin, C.; Lee, B.J. A Systematic Overview of Type II and III Toxin-Antitoxin Systems with a Focus on Druggability. Toxins 2018, 10, 515. [CrossRef]

41. Cook, G.M.; Robson, J.R.; Frampton, R.A.; McKenzie, J.; Przybilski, R.; Fineran, P.C.; Arcus, V.L. Ribonucleases in bacterial toxin-antitoxin systems. Biochim. Biophys. Acta 2013, 1829, 523-531. [CrossRef] [PubMed] 
42. Kang, S.M.; Kim, D.H.; Lee, K.Y.; Park, S.J.; Yoon, H.J.; Lee, S.J.; Im, H.; Lee, B.J. Functional details of the Mycobacterium tuberculosis VapBC26 toxin-antitoxin system based on a structural study: Insights into unique binding and antibiotic peptides. Nucleic Acids Res. 2017, 45, 8564-8580. [CrossRef] [PubMed]

43. Kim, D.H.; Kang, S.M.; Park, S.J.; Jin, C.; Yoon, H.J.; Lee, B.J. Functional insights into the Streptococcus pneumoniae HicBA toxin-antitoxin system based on a structural study. Nucleic Acids Res. 2018, 46, 6371-6386. [CrossRef] [PubMed]

44. Kabsch, W. Xds. Acta Crystallogr. D Biol. Crystallogr. 2010, 66, 125-132. [CrossRef]

45. Adams, P.D.; Afonine, P.V.; Bunkoczi, G.; Chen, V.B.; Davis, I.W.; Echols, N.; Headd, J.J.; Hung, L.W.; Kapral, G.J.; Grosse-Kunstleve, R.W.; et al. PHENIX: A comprehensive Python-based system for macromolecular structure solution. Acta Crystallogr. D Biol. Crystallogr. 2010, 66, 213-221. [CrossRef]

46. Emsley, P.; Lohkamp, B.; Scott, W.G.; Cowtan, K. Features and development of Coot. Acta Crystallogr. D Biol. Crystallogr. 2010, 66, 486-501. [CrossRef]

47. Brunger, A.T. Free R value: A novel statistical quantity for assessing the accuracy of crystal structures. Nature 1992, 355, 472-475. [CrossRef]

48. Murshudov, G.N.; Skubak, P.; Lebedev, A.A.; Pannu, N.S.; Steiner, R.A.; Nicholls, R.A.; Winn, M.D.; Long, F.; Vagin, A.A. REFMAC5 for the refinement of macromolecular crystal structures. Acta Crystallogr. D Biol. Crystallogr. 2011, 67, 355-367. [CrossRef]

49. Chen, V.B.; Arendall, W.B.; Headd, J.J.; Keedy, D.A.; Immormino, R.M.; Kapral, G.J.; Murray, L.W.; Richardson, J.S.; Richardson, D.C. MolProbity: All-atom structure validation for macromolecular crystallography. Acta Crystallogr. D Biol. Crystallogr. 2010, 66, 12-21. [CrossRef]

50. UniProt, C. UniProt: A worldwide hub of protein knowledge. Nucleic Acids Res. 2019, 47, D506-D515. [CrossRef]

51. Thompson, J.D.; Higgins, D.G.; Gibson, T.J. CLUSTAL W: Improving the sensitivity of progressive multiple sequence alignment through sequence weighting, position-specific gap penalties and weight matrix choice. Nucleic Acids Res. 1994, 22, 4673-4680. [CrossRef] [PubMed]

52. Robert, X.; Gouet, P. Deciphering key features in protein structures with the new ENDscript server. Nucleic Acids Res. 2014, 42, W320-W324. [CrossRef] [PubMed] 